\title{
«NowcRadiation» — Software de nowcasting para la predicción de la radiación solar
}

https://doi.org/10.31978/639-19-010-0.061

\author{
Mauri Martínez Sánchez1 (mmartinezs@aemet.es) \\ Antonio Rodríguez Martínez² (arodriguezm@aemet.es) \\ José Antonio García-Moya Zapata33 (jgarciamoyaz@aemet.es) \\ ${ }^{1}$ AEMET / Delegación Territorial en Cataluña / Grupo de Predecibilidad \\ ${ }^{2}$ AEMET / Departamento de Desarrollo y Aplicaciones / Área de Innovación \\ ${ }^{3}$ AEMET / Departamento de Desarrollo y Aplicaciones / Área de Innovación (jubilado)
}

\begin{abstract}
RESUMEN
NowcRadiation es el software desarrollado por AEMET para dar respuesta a la petición de Red Eléctrica de España (REE) y que proporciona cada 15 minutos predicciones de las acumulaciones horarias de las radiaciones solares global horizontal (GHI) y directa normal (DNI) para las siguientes 4 horas con intervalos de 15 minutos, siempre para emplazamientos puntuales. NowcRadiation se fundamenta en la combinación de las radiaciones solares calculadas teóricamente a partir de parámetros astronómicos y modificadas de acuerdo con productos de nubosidad basados en el producto High Resolution Winds EUMETSAT SAFNWC/MSG, del producto Cloud Type de EUMETSAT SAFNWC/MSG y de la predicción de radiación solar del modelo de alta resolución HARMONIE/AROME.

La utilidad de estas predicciones para REE es la de anticipar cambios bruscos en la generación de energía a partir de plantas solares y poder así tomar las acciones correctivas necesarias para asegurar el suministro eléctrico continuo en toda España. Este es uno de los aspectos más importantes para poder incluir fuentes de energía dependientes de las condiciones meteorológicas (eólicas y solares) en el sistema de generación español.
\end{abstract}

PALABRAS CLAVE: predicción numérica; radiación solar; nowcasting; satélite; NWP.

\section{INTRODUCCIÓN}

En enero de 2015 el Área de Innovación (Departamento de Aplicaciones y Desarrollo) de AEMET comenzó a desarrollar un software para la previsión de la radiación global y directa en emplazamientos solares a muy corto plazo. El proyecto se inicia debido al interés que muestra Red Eléctrica de España (REE) ante la posibilidad de incluir el paquete de software SAFNWC/MSG en la predicción de la radiación solar directa y global, dependiente de la nubosidad, en diferentes emplazamientos de plantas solares en la geografía española.

Se trata de un proyecto interdepartamental donde están involucradas varias áreas de AEMET, además del Área de Innovación:

- Satellite Application Facility for support to Nowcasting (SAFNWC): es un proyecto de EUMETSAT liderado por AEMET, cuyo objetivo es el uso de las imágenes de satélite en la previsión inmediata y a muy corto plazo. Utilizaremos algunos de los productos derivados de Meteosat Segunda Generación MSG).

- Área de Modelización: salidas del modelo de área limitada HARMONIE-AROME disponible en AEMET. 
- Red Radiométrica Nacional: observaciones de radiación directa normal y global horizontal.

- Sistemas: soporte técnico de software y hardware.

El objetivo general era diseñar, validar y poner operativa una herramienta que en intervalos de 15/30 minutos facilitase predicción de acumulaciones horarias de radiación solar directa normal (DNI) y global horizontal (GHI) para las siguientes 4 horas, con una resolución temporal de 15 minutos. De modo que el software utiliza los datos de satélite (SAF de nowcasting) durante la primera hora de predicción, los proporcionados por el modelo de alta resolución, disponible en AEMET, HARMONIE-AROME, a partir de la cuarta hora, y una combinación de ambas predicciones según una función de transferencia en las horas intermedias.

Para calcular las acumulaciones horarias de radiación utilizaremos dos productos de satélite el Cloud Type (CT) y el EXIM del SAF; este último utiliza los vientos de alta resolución del software para extrapolar cinemáticamente el tipo de nube (CT) observado desde quince minutos hasta cuatro horas.

Extrae las predicciones de las radiaciones acumuladas directa y global horizontales del modelo en superficie, transformando la radiación directa horizontal a normal dividiendo por el coseno del ángulo cenital.

\section{BUSCANDO UNA FUNCIÓN DE TRANSICIÓN}

Simulando condiciones operativas durante el periodo del 1 de diciembre de 2016 al 15 de junio de 2017 en las estaciones de Arenosillo, Badajoz, Córdoba, Lleida, Madrid, Murcia, Santander y Palma de Mallorca, y entre el 1 de febrero y el 15 de junio de 2017 para Tenerife hemos calculado el error cuadrático medio dividido por la media de la observación, nRMSE, en función del alcance de predicción de las acumulaciones horarias de radiación tanto de los módulos de satélite y modelo para buscar una función de transición que integre ambas predicciones de radiación.

Para la GHI en la figura 1 el nRMSE del módulo del satélite representado por la línea azul continua muestra menores errores que el modelo (línea verde continua) en alcances menores, aunque el nRMSE modelo presenta un comportamiento más estable en todo el rango de predicción. Por lo que deducimos que el módulo del satélite proporcionará mejores predicciones en los primeros tramos de predicción y el modelo en los últimos. El valor de BIAS normalizado por la media de la observación, nBIAS, del satélite representado por la línea azul punteada es negativo y disminuye con el alcance, mientras que el del modelo es positivo y se muestra más estable a lo largo de todo el rango de predicción.

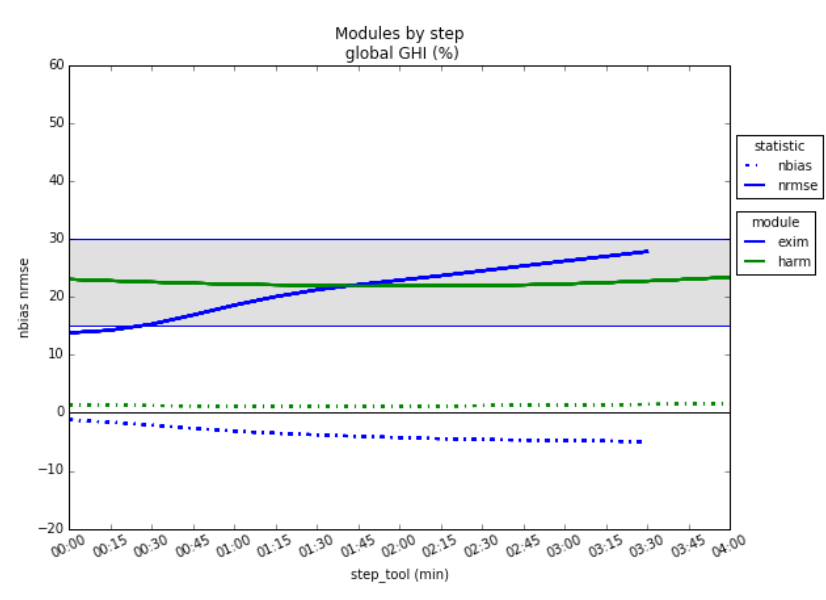

Figura 1. nRMSE y nBIAS de las acumulaciones horarias de GHI de los módulos de satélite, línea azul, y del modelo, línea verde, en función de los alcances de predicción.

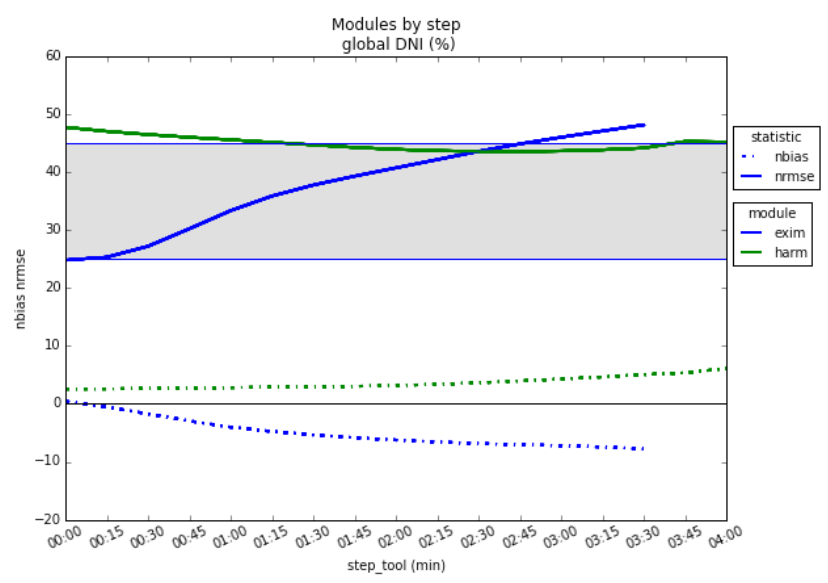

Figura 2. nRMSE y nBIAS de las acumulaciones horarias de DNI de los módulos de satélite, línea azul, y del modelo, línea verde, en función de los alcances de predicción. 
Al igual que sucedía con la GHI, para el caso de la DNI, en la figura 2, el nRMSE del módulo de satélite es menor en alcances más bajos aunque en este caso las líneas de errores normalizados de los módulos de satélite y del modelo se cortan en un alcance mayor al caso de la GHI, lo que nos indicaría que el satélite podría tener más peso hasta mayores alcances, el comportamiento del modelo sigue siendo más estable con todos los alcances. El nBIAS muestra un comportamiento idéntico al que mostraba el de la GHI.

Nuestro objetivo es combinar las predicciones de radiación acumulada dada por los módulos de satélite y modelo de modo que su nRMSE esté dentro del rango de errores esperados para satélites en regiones nubosas y latitudes medias que se describe en Pérez et al. (2013). Para el caso de la GHI este rango es del 15-30\%, mientras que para el caso de la DNI este rango es del 25-45 \%, en las gráficas se representa por el área sombreada.

En la figura 3 representamos los nRMSE de las predicciones de acumulaciones horarias de GHI en función del alcance, para distintas funciones. La función tangente hiperbólica centrada en el horizonte de 120 minutos, curva cian, muestra el comportamiento más abrupto de todas ellas por lo que será descartada. Las cuadráticas en descenso (curvas verdes) muestran errores más bajos en alcances bajos y errores más altos en los alcances altos, comportamiento opuesto al mostrado por las cuadráticas en ascenso (curvas azules). Los errores más bajos son los que muestran las de tipo lineal (curvas rojas) mostrando una transición más suave al aumentar el rango de transición entre los dos módulos.

Las mismas funciones aplicadas a la DNI muestran un comportamiento parecido; en la figura 4, las transiciones de tipo lineal representadas por las curvas rojas son las que presentan menor valor de nRMSE y una transición más suave a medida que se amplía el intervalo de transición.

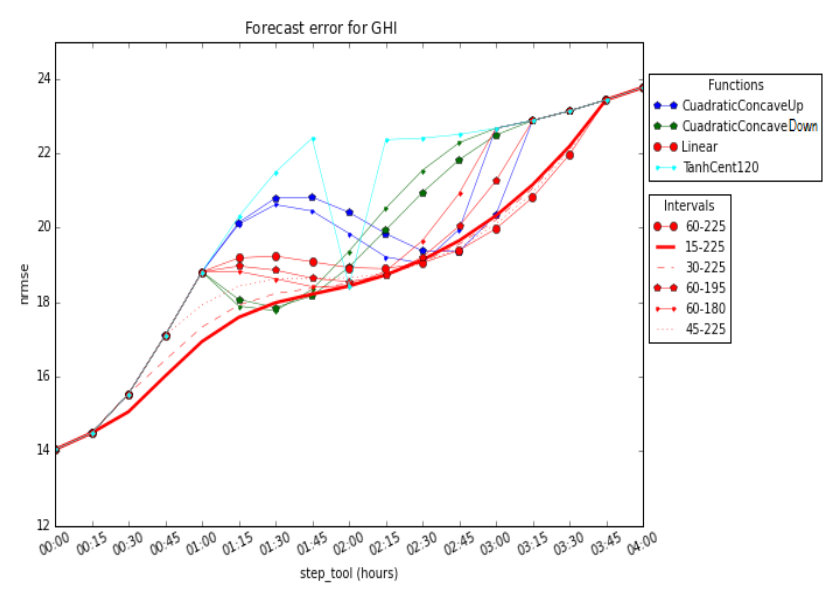

Figura 3. nRMSE de las distintas funciones de transición para la GHI.

En vista de los resultados se opta por una función lineal, para ambas radiaciones, por ser la que proporciona menores valores de nRMSE, la cual toma el valor 0 de predicción del modelo a los 15 minutos y el valor 1 a los 225 minutos. Su distribución de pesos puede verse en la figura 5 .

Figura 5.

Representación gráfica de la distribución de pesos de la función de transición tipo lineal.

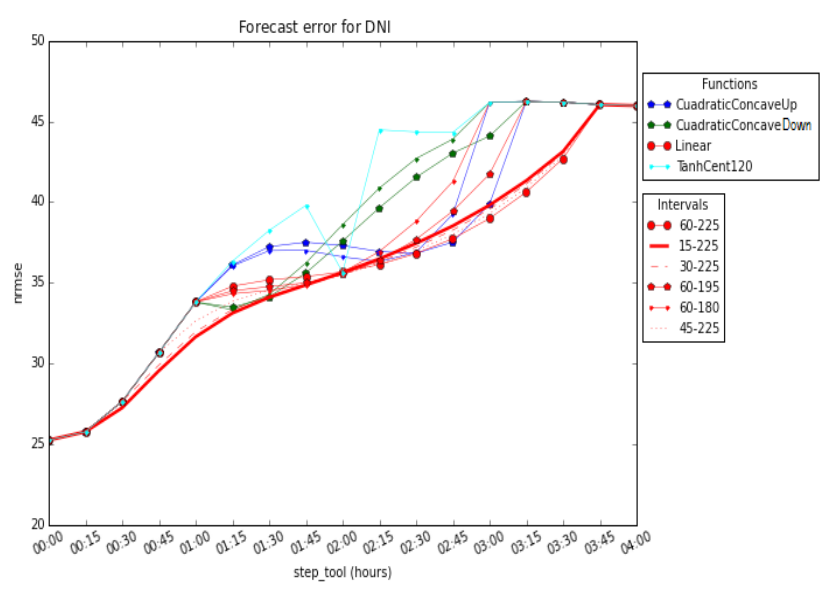

Figura 4. nRMSE de las distintas funciones de transición para la DNI.

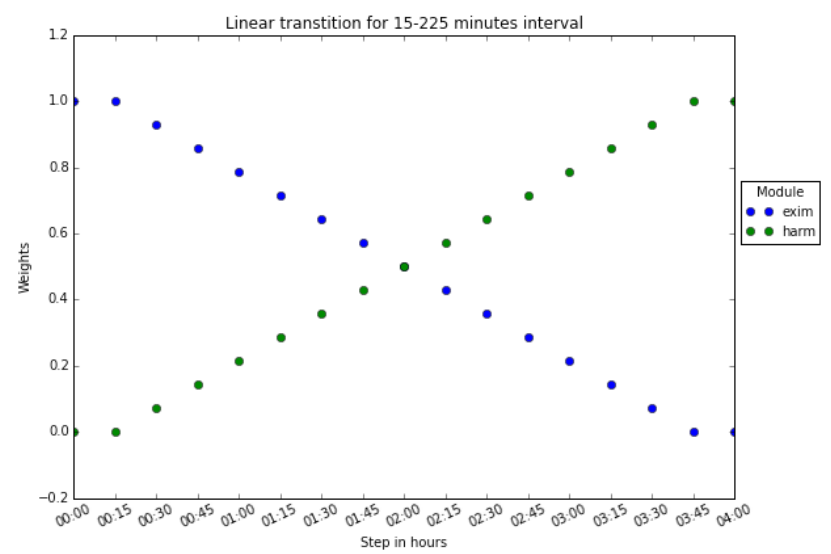




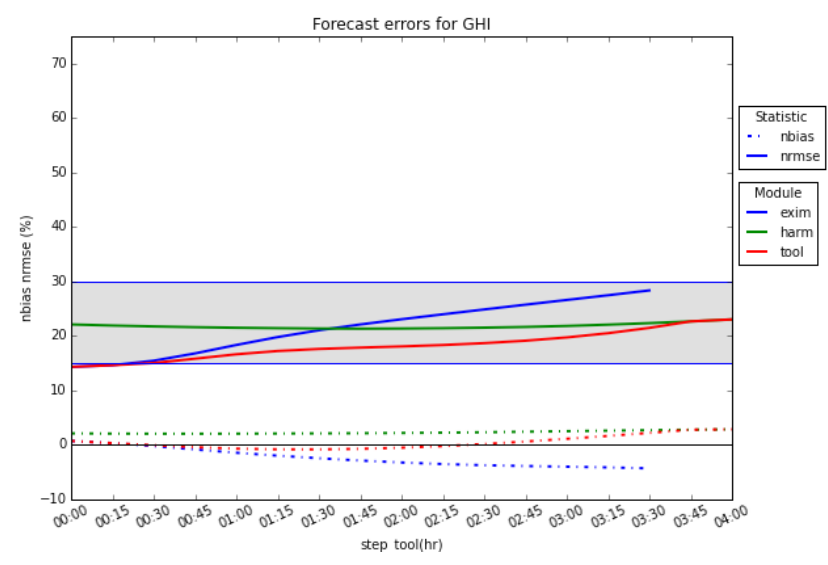

Figura 6. nRMSE y nBIAS de las predicciones de radiación acumulada horaria de la GHI para los módulos de satélite (curva azul), modelo (curva verde) y herramienta (línea roja) en función del alcance de la predicción.

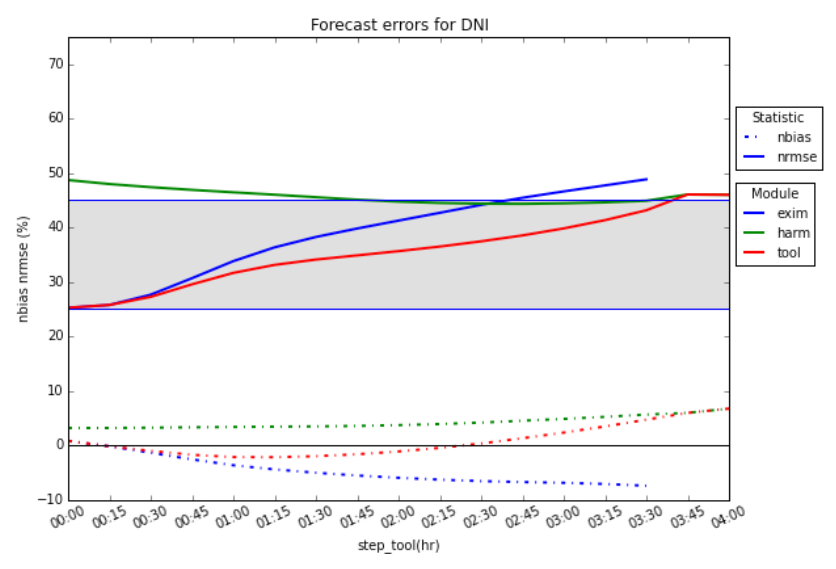

Figura 7. nRMSE y nBIAS de las predicciones de radiación acumulada horaria de la DNI para los módulos de satélite (curva azul), modelo (curva verde) y herramienta (línea roja) en función del alcance de la predicción.

En la figura 6 se muestra la función de transición elegida en el caso concreto de la GHI; se observa que el nRMSE proporcionado por la herramienta - línea roja continua - se encuentra dentro del área sombreada que representa el rango de valores esperados en predicciones con satélite para regiones nubosas y latitudes medias. Además es siempre menor que el proporcionado por los módulos de satélite — línea azul continuao modelo — línea verde continua_- esto es debido a que la herramienta compensa los nBIAS — líneas punteadas- del satélite y del modelo.

El nRMSE de las predicciones de radiación acumulada a una hora para la DNI presenta, en la figura, un comportamiento similar al de la GHI descrito anteriormente, salvo que el último tramo de la predicción se encuentra fuera del rango de valores esperados para las predicciones de satélite en regiones nubosas y latitudes medias, aunque sí se encuentra dentro del rango de valores de errores esperados para modelos mesoescalares a 24 horas que es de 30-100\% para la DNI, tal y como se indica en LARA FANEGO et al., 2012.

\section{COMPORTAMIENTO MENSUAL POR LOCALIZACIÓN}

En la figuras 8 y 9 se muestran los valores del nRMSE y nBIAS de predicciones de radiación acumulada horaria para diferentes ubicaciones de la península ibérica y Canarias dadas por la herramienta, simulando

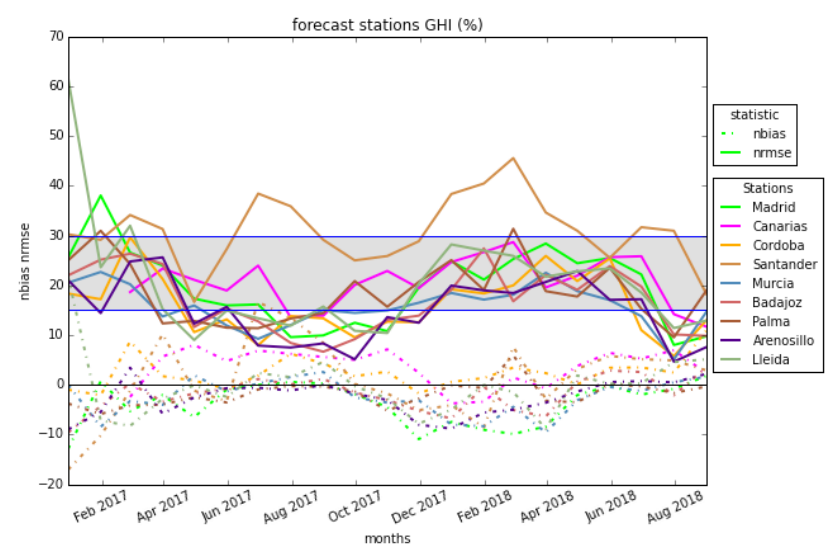

Figura 8. nRMSE y nBIAS de las predicciones de radiación acumulada GHI para diferentes estaciones a lo largo del periodo 1 de diciembre de 2016 al 31 de agosto de 2018.

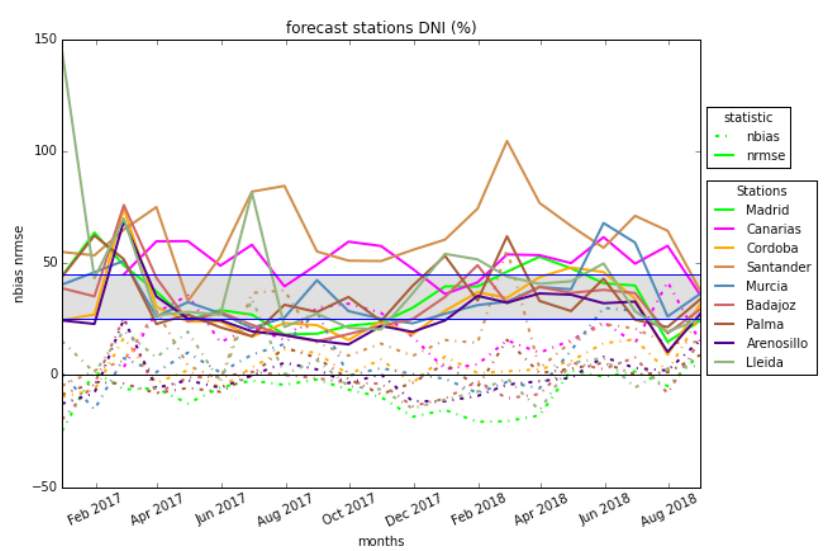

Figura 9. nRMSE y nBIAS de las predicciones de radiación acumulada DNI para diferentes estaciones a lo largo del periodo 1 de diciembre de 2016 al 31 de agosto de 2018. 
condiciones operativas. En general ambas predicciones muestran un comportamiento estacional, quedando la mayoría de las estaciones en los rangos esperados de predicciones por satélite, áreas sombreadas, aunque el comportamiento es diferente en función de su posición geográfica. Las estaciones con latitudes mayores presentan los peores resultados.

\section{NOWCRADIATION}

El software nowcRadiation diseñado en AEMET proporciona cada 15 minutos acumulaciones horarias de radiación global horizontal y directa normal con alcances cada 15 minutos para las siguientes cuatro horas. Las acumulaciones horarias de las figuras siguientes vienen dadas en (julios/metro cuadrado)/3600 segundos, es decir, en watios/metro cuadrado.

\subsection{Cielo despejado}

Las predicciones de radiación acumuladas a una hora para la GHI a lo largo del día 8 de septiembre de 2018 en Arenosillo (Huelva) se representan en la figura 10, cada curva representa una predicción de 4 horas; la figura 11 muestra una comparación visual de la predicción del software (curvas rojas) con la observación (línea cian). La curva de predicción se asemeja a la de observación quedando ligeramente por debajo, lo que nos indica que se han subestimado las predicciones de radiación.

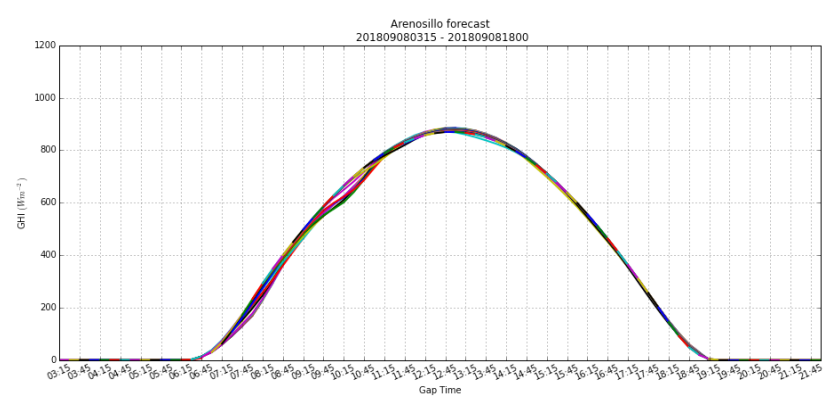

Figura 10. Predicciones de la radiación acumulada a una hora de GHI para el Arenosillo a lo largo del día

8 de septiembre de 2018 proporcionada por el software nowcRadiation.

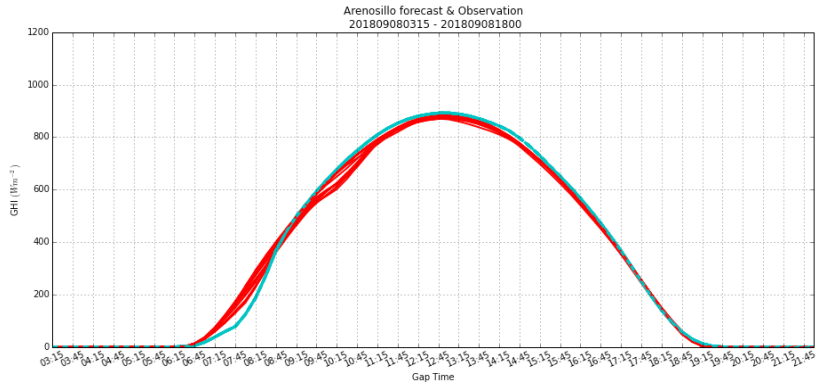

Figura 11. Comparación visual de las salidas del software nowcRadiation (en rojo) con la observación (cian) para el día 8 de septiembre de 2018 en el Arenosillo.

En la figura 12 cada curva representa la predicción a 4 horas de la predicción de acumulaciones horarias de DNI a lo largo del día 10 de septiembre de 2018 en Córdoba; la figura 13 es una comparación visual de las predicciones dadas por nowcRadiation (curvas rojas) y la observación (curva cian). Las curvas de predicción tienen la misma forma que la de observación quedando ligeramente desplazadas hacia arriba, lo que nos indica una sobreestimación de la predicción de radiación.

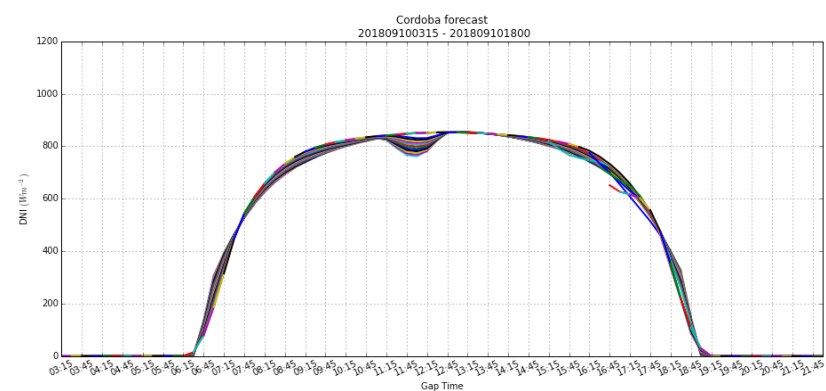

Figura 12. Predicciones de la radiación acumulada a una hora de DNI para Córdoba a lo largo del día 10 de septiembre de 2018 proporcionada por el software nowcRadiation.

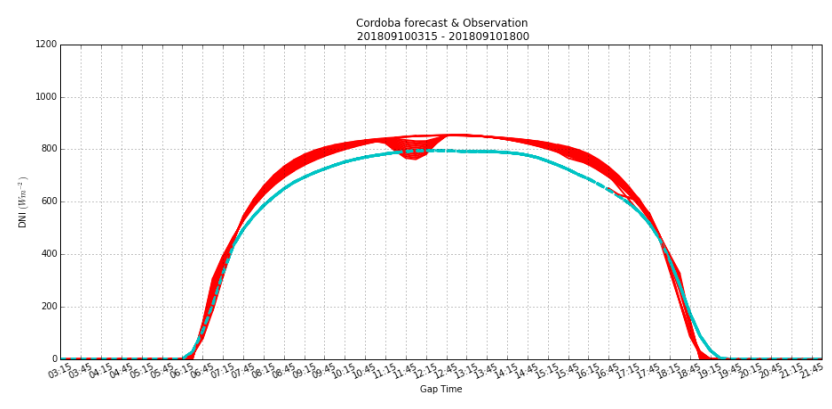

Figura 13. Comparación visual de las salidas del software nowcRadiation (en rojo) con la observación (cian) para el día 10 de septiembre de 2018 en Córdoba. 


\subsection{Cielo cubierto}

Hemos visto el comportamiento con cielo despejado; el comportamiento con días nubosos se aprecia en las figuras 14 y 15 para predicciones de GHI y DNI comparadas con la observación, que en alcances bajos el software es capaz de distinguir la nube y seguir la evolución.

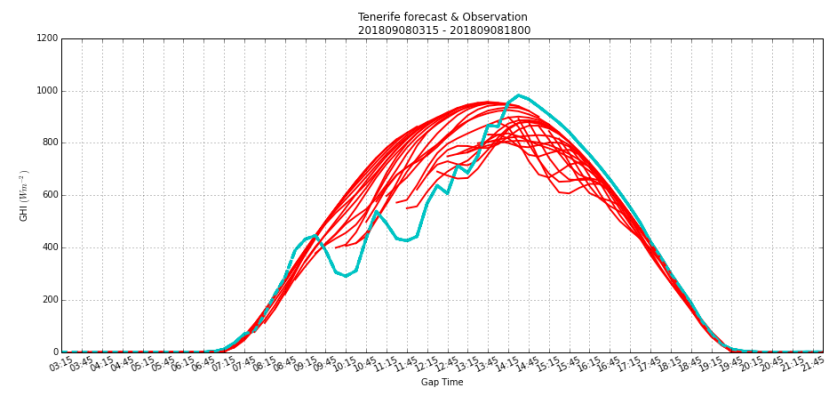

Figura 14. Comparación visual de las salidas del software nowcRadiation, predicción de la radiación acumulada GHI (en rojo), con la observación (cian), para el día 8 de septiembre de 2018 en Tenerife, ejemplo de día nuboso.

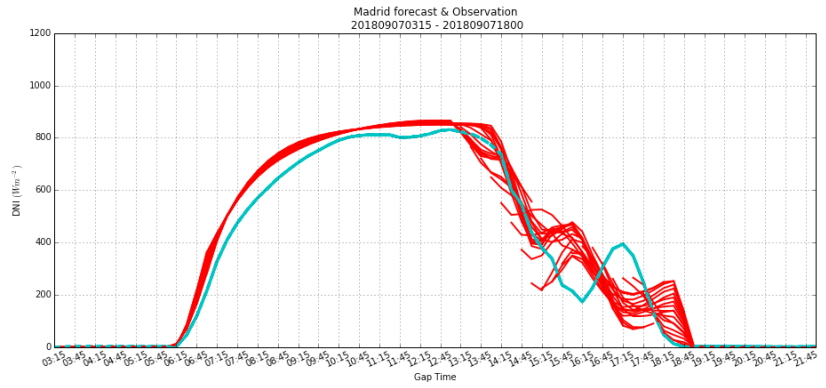

Figura 15. Comparación visual de las salidas del software nowcRadiation, predicción de la radiación acumulada DNI (en rojo), con la observación (cian), para el día 7 de septiembre de 2018 en septiembre, ejemplo de día nuboso.

\section{MAS INFORMACIÓN}

Más información sobre este proyecto en:

- Rodríguez Martínez, A. y Martínez Sánchez, M., 2016. Predicción inmediata y a muy corto plazo de la radiación solar a partir de datos de satélite y modelos numéricos. http://www.iic.uam.es/energias/prediccion-radiacion-solar-corto-plazo/.

- EMS Annual Meeting: European Conference for Applied Meteorology and Climatology 2017 (04-07 Sept 2017), Dublin, Ireland.

Session Operational Systems and Applications — Energy Meteorology.

Resumen: https://meetingorganizer.copernicus.org/EMS2017/EMS2017-128.pdf.

Póster: https://presentations.copernicus.org/EMS2017-128\_presentation.pdf.

\section{CONCLUSIONES}

Desde enero de 2015 el Área de Innovación del Departamento de Desarrollo y Aplicaciones de AEMET ha desarrollado nowcRadiation, una herramienta de nowcasting para la predicción de la radiación solar directa y global en emplazamientos de plantas solares mediante contrato mantenido durante dos años (2015-2017) entre REE y AEMET. La herramienta final facilita en intervalos de 15 minutos la predicción de los valores horarios de la radiación solar directa normal (DNI) y global horizontal (GHI) acumuladas a una hora para las siguientes 4 horas, con una resolución temporal de 15 minutos. Para ello se utilizan observaciones y predicciones del tipo de nube calculados utilizando información del satélite MSG y predicciones de la radiación en superficie del modelo numérico de alta resolución HARMONIE-AROME disponible en AEMET.

\section{MóduloSAF}

La herramienta utiliza la información del tipo de nube (CT) generada cada 15 minutos por el software SAFNWC/MSG. Para cada categoría nubosa se ha obtenido una función de ajuste entre la radiación observada en siete estaciones peninsulares pertenecientes a la Red Radiométrica Nacional de AEMET y el ángulo cenital solar. Los mejores resultados se han obtenido utilizando funciones de tipo lineal para la GHI y tipo 
cúbico para la DNI. Una vez obtenidos los valores instantáneos de la irradiancia solar a partir del CT se calculan las acumulaciones horarias utilizando una integración trapezoidal de los valores correspondientes a la hora anterior. El producto EXIM del SAF utiliza los vientos de alta resolución del software para extrapolar cinemáticamente el tipo de nube observado desde 15 minutos hasta cuatro horas.

\section{Módulo HARMONIE-AROME}

La herramienta extrae las predicciones de la radiación acumulada horaria directa global GHI y directa horizontal DHI en superficie del modelo HARMONIE-AROME integrado en AEMET en dos dominios incluyendo la península ibérica y Canarias.

El modelo se integra cada 3 horas con una resolución horizontal de 2,5km y horizonte de predicción de $+00 \mathrm{~h}$ a +48 h y salidas cada 15 minutos. La radiación DHI se convierte en DNI dividiendo por el coseno del ángulo cenital.

\section{NowcRadiation}

La herramienta de nowcasting proporciona cada 15 minutos los valores de las acumulaciones horarias GHI y DNI para las 4 horas siguientes con alcances cada 15 minutos. Las predicciones SAF y HARMONIE-AROME se combinan según pesos proporcionados por una función de transición dependiendo del horizonte temporal.

Se han estudiado varios tipos de funciones de transición y se ha elegido, por proporcionar menores errores en la predicción, una función lineal que toma el valor 0 para la predicción del modelo a los 15 minutos y 1 a los 225 minutos. La suma de los pesos de ambos módulos es uno siendo dominante la contribución del satélite las dos primeras horas y la del modelo las dos últimas.

Se han analizado los errores de predicción de la herramienta comparando los valores previstos de la GHI y DNI con los observados en 10 estaciones de la Red Radiométrica Nacional de AEMET, 7 peninsulares y 3 insulares. Como resumen de los estudios de validación destacamos:

- Globalmente, considerando todas las estaciones, el satélite proporciona mejores predicciones en los primeros tramos de la predicción y el modelo al final, como estaba previsto en los primeros planteamientos del proyecto.

- Globalmente, la predicción del satélite presenta un bias negativo que aumenta con el horizonte temporal mientras que la predicción del modelo presenta valores positivos aumentando con el horizonte para la DNI y más estables para la GHI. La herramienta compensa ambos comportamientos presentando siempre menores errores de predicción que cada módulo por separado en base al RMSE.

- Los resultados de validación de la herramienta, en términos de valores normalizados del RMSE, se encuentran - para el conjunto de las estaciones — dentro del rango de errores esperados de los modelos satelitales, tanto para la GHI (en todos los tramos previstos) como para la DNI (salvo para el último tramo). Destacar la ventaja de que el componente satelital de la herramienta no requiere de la utilización de un modelo de transferencia radiativa como ocurre con los modelos clásicos de satélite.

- En los estudios de validación por estaciones se encuentra que las predicciones para estaciones peninsulares por debajo de los $40^{\circ}$ de latitud y la insular de Palma de Mallorca presentan mejores resultados que los encontrados en Canarias. De igual forma se presenta un comportamiento diferente según el tipo de nube, empeorando en general con el espesor o capacidad de bloqueo de la radiación de la nube.

En la actualidad el código de la herramienta se está optimizando para su puesta en operación en el Servicio de Explotación de AEMET y terminando la documentación técnica para su posterior uso operativo.

El siguiente paso a seguir será introducir la predicción probabilística en la herramienta. Para ello se utilizarían ensembles de diferentes modelos numéricos de predicción y se aplicaría algún tipo de perturbación a las predicciones de satélite. 


\section{AGRADECIMIENTOS}

Agradecer a Red Eléctrica de España (REE) por creer en este proyecto.

Desde aquí agradecer a todas las áreas de AEMET involucradas en este proyecto su apoyo. A todos los compañeros que nos han facilitado el desarrollo de nowcRadiation, igracias, porque de vosotros también es nowcRadiation! En especial a Alfons Callado Pallarés por su apoyo en estos últimos alcances de predicción y por dejarnos terminarlo.

\section{REFERENCIAS}

Pérez, R., Cebecauer, T. y Suri, M., 2013. Semi-Empirical Satellite Models. En: Solar Resource Assesment and Forecasting. Elsevier.

Lara Fanego, V., Ruiz Arias, J. A., Pozo Vázquez, D., Santos Alamillos, F. J. y Tovar Pescador, J., 2012. Evaluation of the WRF model solar irradiance forecasts in Andalusia (southern Spain). Sol. Energy, 86, 2200-2217, doi:10.1016/j.solener.2011.02.014. 